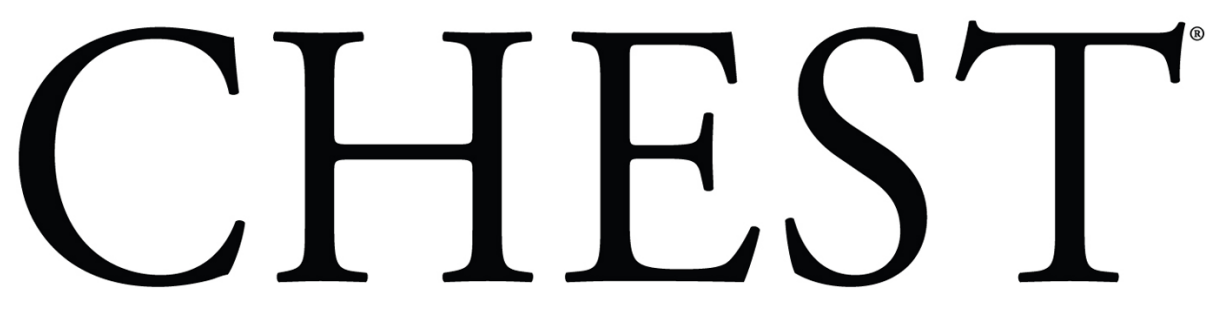

Official publication of the American C ollege of Chest Physicians

Relationship Between Upper Airway and Inspiratory Pump Muscle Force in Obstructive Sleep Apnea

Kelly L. Shepherd, Cathryn M. Jensen, Kathleen J. Maddison, David R. Hillman and Peter R. Eastwood

Chest 2006;130;1757-1764

DOI 10.1378/chest.130.6.1757

The online version of this article, along with updated information and services can be found online on the World Wide Web at: http://chestjournal.org/cgi/content/abstract/130/6/1757

CHEST is the official journal of the American College of Chest Physicians. It has been published monthly since 1935. Copyright 2007 by the American College of Chest Physicians, 3300 Dundee Road, Northbrook IL 60062. All rights reserved. No part of this article or PDF may be reproduced or distributed without the prior written permission of the copyright holder

(http://www.chestjournal.org/misc/reprints.shtml). ISSN: 0012-3692.

A M E R I A N C O L L E G E O F

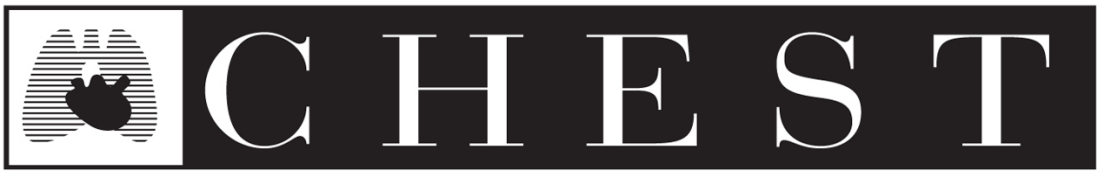
$\begin{array}{llllllllll}\mathrm{P} & \mathrm{H} & \mathrm{Y} & \mathrm{S} & \mathrm{I} & \mathrm{C} & \mathrm{I} & \mathrm{A} & \mathrm{N} & \mathrm{S}^{\circledR}\end{array}$ 


\title{
Relationship Between Upper Airway and Inspiratory Pump Muscle Force in Obstructive Sleep Apnea*
}

\author{
Kelly L. Shepherd, BSc(Hons); Cathryn M. Jensen, BSc; \\ Kathleen J. Maddison, BSc(Hons); David R. Hillman, MD; and \\ Peter R. Eastwood, PhD
}

\begin{abstract}
Background: Upper airway (UA) patency during inspiration is determined by the balance between dilating forces generated by UA dilator muscle activity and collapsing forces related to the decreased intraluminal pressure that accompanies flow generated by inspiratory muscle activity. It is possible that the relative strengths of UA dilator and inspiratory pump muscles could be an important determinant of the susceptibility to UA collapse during sleep (ie, obstructive sleep apnea [OSA]).

Methods: Measurements of tongue protrusion (TP) force and maximum inspiratory pressure (PImax) were obtained in 94 patients admitted for overnight polysomnography for suspected OSA, quantified by apnea-hypopnea index (AHI).

Results: There was a direct linear relationship between TP force and PImax $\left(r^{2}=0.37, \mathrm{p}<0.001\right)$. A high ratio of TP force to PImax (greater than group 90th percentile, $0.027 \mathrm{~kg} / \mathrm{cm} \mathrm{H}_{2} \mathrm{O}$ ) appeared to protect against OSA, as moderate-to-severe OSA (AHI $>20 / \mathrm{h}$ ) was not observed in any individual with a ratio above this threshold. AHI was not linearly related to TP force, PImax, or the ratio of TP force to PImax.

Conclusions: UA muscle strength is linearly related to inspiratory pump muscle strength. The ratio of UA muscle strength (TP force) and inspiratory pump muscle strength (PImax) was not different between individuals with and without OSA; however, a high wakeful ratio of TP force to PImax appears to be associated with a reduced propensity to moderate-to-severe OSA.
\end{abstract}

(CHEST 2006; 130:1757-1764)

Key words: collapsibility; diaphragm; genioglossus; obstructive sleep apnea; respiratory muscle strength; tongue protrusion; upper airway

Abbreviations: $\mathrm{AHI}=$ apnea-hypopnea index; $\mathrm{BMI}=$ body mass index; $\mathrm{FRC}=$ functional residual capacity; OSA = obstructive sleep apnea; PImax = maximum inspiratory pressure; PImax-FRC $=$ maximum inspiratory pressure measured at functional residual capacity; PImax-RV = maximum inspiratory pressure measured at residual volume; $\mathrm{RV}=$ residual volume; $\mathrm{TP}=$ tongue protrusion; $\mathrm{UA}=$ upper airway

$\mathbf{O}$

bstructive sleep apnea (OSA) is characterized by repetitive collapse of the upper airway (UA) during sleep. The tendency for the UA to collapse during sleep is related to the balance between dilating forces generated by UA dilator muscle activity and collapsing forces associated with the intraluminal pressure reductions that accompany flow generated by inspiratory muscle activity. ${ }^{1}$ Hence, it is possible that the relative strength of UA dilator and inspiratory pump muscles could be an important determinant of susceptibility to OSA. While substantial weakness of pharyngeal dilator muscles predisposes to OSA, as evident in neuromuscular disease, ${ }^{2}$ it is unknown whether a predisposition also exists in individuals in whom pharyngeal muscle dilator forcegenerating capacity is more subtly reduced relative to force-generating capacity of the inspiratory muscles. If so, such a predisposition may be evident from measures of the relative strength of these muscles.

Furthermore, it is unclear whether OSA itself influences UA dilator muscle strength. Abnormal structural changes including an increase in type IIa muscle fibers in UA muscles have been reported in individuals with OSA. ${ }^{3-5}$ These changes are consistent with an increased capacity for the UA muscles to produce force. ${ }^{3}$ Alternatively, other structural 
changes, possibly caused by inflammatory processes resulting from snoring-related vibration of pharyngeal walls ${ }^{6}$ could decrease the capacity of the UA muscles to produce force. Functional studies provide conflicting findings with measures of in vitro twitch tension of UA muscle obtained from patients with OSA being either greater than nonapneic snorers, ${ }^{3}$ or not different from normal control subjects. ${ }^{5}$ The force developed during maximal voluntary tongue protrusion (TP) provides an index of functional strength of the genioglossus muscle, the major dilator muscle in the UA, as well as other intrinsic tongue muscles. The only study ${ }^{7}$ to measure TP force in OSA reported a weak negative relationship between TP force and severity of OSA, a finding attributed to an age-related decrease in TP force and increase in severity of OSA.

It is also possible that OSA affects the forcegenerating capacity of inspiratory muscles either directly or because of coexisting obesity that is commonly present in individuals with OSA. Previous studies have reported impaired ventilatory capacity in obese subjects compared to normal individuals, ${ }^{8,9}$ most likely as a consequence of reduced chest wall compliance. ${ }^{10}$ Conversely, inspiratory muscle strength could be preserved or even increased in OSA, as a consequence of the training effect of exaggerated inspiratory efforts made repeatedly throughout sleep against a narrowed or obstructed UA.

The ratio of maximum TP force to maximum inspiratory pressure (PImax) provides a simple index

*From the West Australian Sleep Disorders Research Institute (Ms. Shepherd, Ms. Jensen, Ms. Maddison, and Dr. Hillman), Department of Pulmonary Physiology, Sir Charles Gairdner Hospital, Nedlands, Western Australia; and School of Anatomy and Human Biology (Dr. Eastwood), University of Western Australia, Perth, Australia.

This article was submitted through the West Australian Sleep Disorders Research Institute, Department of Pulmonary Physiology, Sir Charles Gairdner Hospital, Nedlands, Western Australia.

Ms. Shepherd, Dr. Eastwood, and Dr. Hillman contributed to the study conception, design, data interpretation, and preparation and revision of the manuscript. Ms. Shepherd, Ms. Jensen, and Ms. Maddison were primarily responsible for data collection.

This study was funded, in part, by National Health and Medical Research Council (Australia) Grant No. 303218. Dr. Eastwood was supported by a National Health and Medical Research Council (Australia) R. Douglas Wright Fellowship (No. 294404). The authors have no conflict of interests with any part of this study to disclose.

Manuscript received December 9, 2005; revision accepted June 13, 2006.

Reproduction of this article is prohibited without written permission from the American College of Chest Physicians (www.chestjournal. org/misc/reprints.shtml).

Correspondence to: Peter R. Eastwood, PhD, West Australian Sleep Disorders Research Institute, Internal Mailbox 201, Queen Elizabeth II Medical Centre, Nedlands, Western Australia, 6009; e-mail: Peter.Eastwood@health.wa.gov.au

DOI: $10.1378 /$ chest.130.6.1757 of the relative strength of UA dilator and inspiratory muscles. This study sought to determine whether there was any relationship between the ratio of TP force to PImax and the propensity for OSA. We hypothesized that waking measures of the ratio of UA muscle force to inspiratory pump muscle force would better predict severity of OSA than waking measures of UA muscle force or respiratory pump muscle force alone, such that individuals with greater UA muscle force relative to pump muscle force would be protected from UA collapse during sleep.

\section{Materials AND Methods}

\section{Subjects}

Ninety-four consecutive patients (52 men and 42 women) attending the Respiratory Sleep Disorders Clinic at Sir Charles Gairdner Hospital for overnight polysomnography were recruited into the study (Table 1). Subjects were excluded from participation if they had been or were being treated with continuous positive airway pressure, had undergone previous surgical treatment for sleep-disordered breathing, or if they had neuromuscular or pulmonary disease. This study was approved by the Sir Charles Gairdner Hospital Human Research Ethics Committee. Informed consent was obtained from each subject prior to participation in the study

\section{Protocol}

Measurements of TP force and PImax were obtained during the setup period of each individual's clinical overnight polysom-

Table 1-Subject Characteristics*

\begin{tabular}{|c|c|c|}
\hline Characteristics & Men & Women \\
\hline Subjects, No. & 52 & 42 \\
\hline Age, yr & $47(13)$ & $49(14)$ \\
\hline Height, $\mathrm{cm}$ & $177(7) \uparrow$ & $164(7)$ \\
\hline BMI, $\mathrm{kg} / \mathrm{m}^{2}$ & $31(5)$ & $34(9)$ \\
\hline $\mathrm{AHI}, / \mathrm{h}$ & $30(34)$ & $21(25)$ \\
\hline Neck circumference, mm & $443(42) \uparrow$ & $399(48)$ \\
\hline Cricomental distance, $\mathrm{mm}$ & $68(12)$ & $66(13)$ \\
\hline Cricomental space, $\mathrm{mm}$ & $3.9(4.1)$ & $2.7(4.6)$ \\
\hline PImax-FRC, $\mathrm{cm} \mathrm{H}_{2} \mathrm{O}$ & $109(36) \uparrow$ & $79(26)$ \\
\hline PImax- $\mathrm{RC}, \mathrm{cm} \mathrm{H}_{2} \mathrm{O}$ & $118(35)$ 十克 & $88(24) \stackrel{+}{+}$ \\
\hline PImax-FRC, $\%$ predicted & $104(32) \uparrow$ & $119(35)$ \\
\hline PImax-RV, \% predicted & $100(26)$ & $107(25)$ \\
\hline TP force seated, $\mathrm{kg}$ & $2.0(0.5) \uparrow$ & $1.6(0.4)$ \\
\hline $\mathrm{TP}$ force supine, $\mathrm{kg}$ & $2.1(0.5) \nmid+$ & $1.7(0.5)$ 龺 \\
\hline $\begin{array}{l}\text { Ratio of TP force to PImax-FRC } \\
\text { seated, } \mathrm{kg} / \mathrm{cm} \mathrm{H}_{2} \mathrm{O}\end{array}$ & $0.019(0.006)$ & $0.022(0.010)$ \\
\hline $\begin{array}{l}\text { Ratio of TP force to PImax-FRC } \\
\text { supine, } \mathrm{kg} / \mathrm{cm} \mathrm{H}_{2} \mathrm{O}\end{array}$ & $0.020(0.005)$ & $0.023(0.010)$ \\
\hline $\begin{array}{l}\text { Ratio of TP force to PImax-RV } \\
\text { seated, } \mathrm{kg} / \mathrm{cm} \mathrm{H}_{2} \mathrm{O}\end{array}$ & $0.017(0.005)$ & $0.019(0.007)$ \\
\hline $\begin{array}{l}\text { Ratio of TP force to PImax-RV } \\
\text { supine, } \mathrm{kg} / \mathrm{cm} \mathrm{H}_{2} \mathrm{O}\end{array}$ & $0.018(0.005)$ & $0.020(0.007)$ \\
\hline
\end{tabular}


nography. TP force was measured in the seated and supine postures. PImax was measured with the subjects seated at functional residual capacity (PImax measured at FRC [PImaxFRC]) and residual volume (PImax measured at RV [PImax-RV]). Anthropometric measurements were also obtained at this time, and included height, weight, body mass index (BMI), neck circumference, cricomental distance, and cricomental space.

\section{Measurements}

TP Force: A lingual force transducer was constructed based on the device described by Mortimore et al. ${ }^{11}$ The mouthpiece consisted of a $1.0-\mathrm{cm}$ diameter nylon plate connected to a load cell. Movement of the plate on the load cell was negligible. Behind the nylon plate was a groove $2.0 \mathrm{~mm}$ deep and $2.0 \mathrm{~mm}$ wide. Subjects were asked to rest their upper and lower incisors in the groove while supporting the transducer with one hand, and then to push their tongue as hard as possible against the nylon plate for approximately $2 \mathrm{~s}$. Subjects were encouraged to produce a maximal effort during each attempt. The change in force with time was displayed on an oscilloscope positioned in front of the subject.

Maximum TP force was defined as the peak force generated in three attempts in which the peak force differed by $<5 \%$. In the majority of cases, this required no more than six attempts. Subjects rested for approximately $30 \mathrm{~s}$ between trials. Measurements were obtained in both seated and supine postures with the head in a neutral position (when sitting, they were instructed to look straight ahead; when supine, the head was maintained in a neutral position using a head rest [Shea head rest; Gyrus ENT; Memphis, TN]). The force transducer was connected to an amplifier (Analog Devices; Norwood, MA), and data were recorded at $100 \mathrm{~Hz}$ on a data acquisition and analysis system (Powerlab model 16s; ADInstruments; Sydney, Australia).

PImax: PImax was measured using a noncollapsible mouthpiece. A leak of $1.0 \mathrm{~mm}$ in diameter was created in the mouthpiece to minimize generation of pressure by the facial muscles. Pressure within the mouthpiece was monitored with a pressure transducer (Monitoring Kit; Abbott; Sligow, Ireland) and custom-made amplifier (Medical Technology and Physics, Sir Charles Gairdner Hospital; Nedlands, Western Australia) and recorded at $100 \mathrm{~Hz}$ on a data acquisition and analysis system (Powerlab model 16s).

Subjects were encouraged to perform a maximum inspiratory effort from normal FRC or RV against an occluded mouthpiece. Care was taken to ensure a stable pattern of quiet breathing prior to the FRC measurement and for complete expiration prior to the RV measurements. Each effort was maintained for approximately 2 s. Subjects rested for at least $30 \mathrm{~s}$ between efforts. PImax was defined as the peak pressure generated in three efforts in which the peak pressure differed by $<5 \%$. In most cases, no more than six efforts were required. All measurements were made while the subject was seated with the head in a neutral position.

Polysomnography: Overnight polysomnography was performed using a computerized data acquisition system (E-Series; Compumedics; Melbourne, Australia). This included monitoring of EEG (C4-A1 and C3-A2) with gold cup electrodes attached to the scalp, left and right electrooculograms, submental electromyogram, and ECG recorded with surface electrodes, abdominal and thoracic effort (inductance plethysmography), nasal and oral airflow, nasal pressure, leg movements, oxygen saturation, body position (mercury switch position sensor positioned on patients' chest), and sound intensity measured by microphone suspended above the subject. Sleep stage analysis was performed according to standard criteria. ${ }^{12}$ Respiratory events during sleep were also analyzed according to recognized criteria. ${ }^{13}$ Severity of sleep- disordered breathing was reflected in the apnea-hypopnea index (AHI), which is defined as the number of apneas and hypopneas per hour of sleep. An AHI of 20/h was defined as the cutoff for clinically significant OSA, as an AHI > 20/h has been shown to be associated with significantly greater mortality than an AHI $<20 /$ h. $^{14}$

\section{Data Analysis}

Comparisons of variables between male and female subjects and between subjects with and without OSA were performed using unpaired $t$ tests. Linear regression analyses were used to examine the relationships between TP force, PImax, and AHI. For these analyses, AHI was logarithmically transformed to normalize its distribution. ${ }^{7}$ Predicted values for PImax-FRC and PImax-RV were obtained from those described by Hamilton et $\mathrm{al}^{15}$ and Black and Hyatt. ${ }^{16}$ The $\chi^{2}$ test was used to determine whether the 90th percentile of the ratio of TP force to PImax could be used to identify individuals with and without OSA. When appropriate, TP force and PImax were corrected for age and BMI by dividing through by these variables. Data are presented as mean $\pm \mathrm{SD}$, and $\mathrm{p}<0.05$ was considered significant for all comparisons.

\section{RESULTS}

\section{Subjects}

A total of 94 subjects were studied (Table 1). As a group, they were obese (BMI, $32 \pm 8 \mathrm{~kg} / \mathrm{m}^{2}$ ) and had moderate OSA (AHI, $25 \pm 30 / \mathrm{h}$ ). Compared to female subjects, male subjects were taller and had greater neck circumference. BMI, AHI, neck circumference, and cricomental space were greater in subjects with OSA vs subjects without OSA (Table 2). On average, subjects slept for $5.8 \pm 1.7 \mathrm{~h}$, had an average sleep efficiency of $76.1 \pm 12.6 \%$, and spent $2.3 \pm 3.4 \%$ of the night in stage 1 sleep, $66.8 \pm 12.6 \%$ in stage 2 sleep, $9.2 \pm 6.4 \%$ in stage 3 sleep, $14.6 \pm 11.4 \%$ in stage 4 sleep, and $16.9 \pm 7.3 \%$ in rapid eye movement sleep.

\section{TP Force}

TP force (seated and supine) was greater in male than female subjects (Table 1). TP force measured supine was greater than TP force measured seated both in male and female subjects, and when all subjects were combined. These differences remained when correcting TP force for age and BMI.

TP force measured supine was greater in individuals with OSA than those without OSA (Table 2), but was not different when TP force was corrected for age and BMI. No difference was observed between the groups when TP force was measured seated (Table 2).

No significant relationships were observed between $\log$ AHI and TP force whether measured in the seated or supine postures either for all subjects combined or when separated into those with and 
Table 2-Anthropometric Data and Measures of Muscle Strength for Individuals With and Without OSA*

\begin{tabular}{lcc}
\hline \hline \multicolumn{1}{c}{ Characteristics } & AHI $\leq 20 / \mathrm{h}$ & AHI $>20 / \mathrm{h}$ \\
\hline Subjects (male gender), No. & $53(25)$ & $41(27)$ \\
Age, yr & $46(13)$ & $49(13)$ \\
Height, cm & $170(11)$ & $172(8)$ \\
BMI, kg/m ${ }^{2}$ & $30(7)$ & $36(7) \dagger$ \\
AHI, $\mathrm{h}$ & $8(5)$ & $49(36) \dagger$ \\
Neck circumference, mm & $406(46)$ & $446(45) \dagger$ \\
Cricomental distance, mm & $67(12)$ & $69(12)$ \\
Cricomental space, mm & $4.4(5.0)$ & $2.0(3.0) \dagger$ \\
PImax-FRC, cm $\mathrm{H}_{2} \mathrm{O}$ & $93(33)$ & $104(35)$ \\
PImax-RV, cm $\mathrm{H}_{2} \mathrm{O}$ & $104(30)$ & $110(36)$ \\
PImax-FRC, \% predicted & $107(34)$ & $116(32)$ \\
PImax-RV, \% predicted & $101(26)$ & $104(27)$ \\
TP force seated, kg & $1.7(0.4)$ & $1.9(0.6)$ \\
TP force supine, kg & $1.8(0.5)$ & $2.0(0.5) \dagger$ \\
Ratio of TP force to PImax-FRC & $0.021(0.010)$ & $0.019(0.004)$ \\
$\quad$ seated, kg/cm $\mathrm{H}_{2} \mathrm{O}$ & & \\
Ratio of TP force to PImax-FRC & $0.022(0.010)$ & $0.020(0.005)$ \\
$\quad$ supine, kg/cm $\mathrm{H}_{2} \mathrm{O}$ & & \\
Ratio of TP force to PImax-RV & $0.018(0.006)$ & $0.018(0.004)$ \\
$\quad$ seated, kg/cm $\mathrm{H}_{2} \mathrm{O}$ & & \\
Ratio of TP force to PImax-RV & $0.018(0.006)$ & $0.019(0.005)$ \\
$\quad$ supine, kg/cm $\mathrm{H}_{2} \mathrm{O}$ & & \\
\hline
\end{tabular}

*Data are presented as mean (SD) unless otherwise indicated. $t_{\mathrm{p}}<0.05$ between individuals without OSA $(\mathrm{AHI} \leq 20 / \mathrm{h})$ and with OSA $($ AHI $>20 /$ h).

without OSA (Fig 1). TP force decreased with increasing age in subjects with OSA whether measured seated $\left(r^{2}=0.25, \mathrm{p}<0.01\right)$ or supine $\left(r^{2}=0.17, \mathrm{p}<0.01\right)$. No such relationship was observed in subjects without OSA whether measured seated $\left(r^{2}=0.01, \mathrm{p}=0.473\right)$ or supine $\left(\mathrm{r}^{2}=0.0002\right.$, $\mathrm{p}=0.917)$. No significant relationships were observed between TP force (seated or supine) and BMI either for all subjects combined or when separated into those with and without OSA.

\section{Inspiratory Muscle Strength}

PImax-FRC and PImax-RV were greater in male than female subjects (Table 1). PImax was greater when measured at RV than at FRC in both male and female subjects. These differences remained when PImax was corrected for age and BMI. There were no differences in PImax -FRC or PImax-RV between individuals with and without OSA $(\mathrm{p}=0.067$ and $\mathrm{p}=0.074$, respectively) [Table 2].

No significant relationships were observed between log AHI and PImax-FRC (Fig 2) or PImax-RV in subjects with or without OSA or when all subjects were combined. PImax-FRC and PImax-RV both decreased with increasing age in all subjects whether considered as a single group $\left(r^{2}=0.17, \mathrm{p}<0.001\right.$, or $r^{2}=0.23, \mathrm{p}<0.001$, respectively), or when sep- arated into those with OSA $\left(r^{2}=0.29, \mathrm{p}<0.001\right.$, or $\left.r^{2}=0.36, \mathrm{p}<0.001\right)$ or without OSA $\left(r^{2}=0.14\right.$, $\mathrm{p}=0.007$, or $\left.r^{2}=0.18, \mathrm{p}=0.002\right)$. No significant relationships were observed between PImax and BMI whether measured from FRC or RV.

\section{Ratio}

TP force (seated) was linearly related to PImaxFRC in all subjects whether considered as a single group or as those with and without OSA (Fig 3). Similar relationships were observed between TP force measured supine and PImax-RV (results not shown). These relationships remained significant when TP force (seated and supine) and PImax (FRC and RV) were corrected for age and BMI.

While the ratio of TP force (seated) to PImax-FRC was not linearly related to $\log$ AHI (Fig 4), a threshold effect was observed such that moderateto-severe OSA (AHI > 20/h) was not observed in any individual with a ratio greater than that corresponding to the 90th percentile for the group $(0.027 \mathrm{~kg} / \mathrm{cm}$ $\left.\mathrm{H}_{2} \mathrm{O}\right)\left[\mathrm{p}<0.05, \chi^{2}\right]$. This was also the case for the ratio of TP force to PImax when measured supine and from RV, respectively (results not shown). When the ratio of TP force to PImax was controlled for age and BMI, a significant threshold effect remained between TP force (seated or supine) and PImax (FRC and RV), such that moderate-to-severe OSA was not observed in any individuals with a ratio greater than that corresponding to the 90th percentile for the group. No relationships were observed between the ratio of TP force to PImax and duration of apneas or hypopneas, mean oxygen saturation overnight, or arousal index.

\section{DisCUSSION}

This study measured the strength of UA dilator and inspiratory pump muscles in individuals with and without OSA, and examined the potential for measurements of the relative force-generating capacity of these muscles to identify individuals who may be susceptible to UA collapse during sleep (ie, OSA). The major findings of the study were as follows: (1) in both male and female subjects and when all subjects were considered as a single group, TP force was greater when measured supine than seated; (2) supine TP force was greater in individuals with OSA than without OSA, but not when TP force was corrected for age and BMI; (3) PImax was not different between individuals with and without OSA; (4) UA muscle strength (TP force) was linearly related to inspiratory pump muscle strength; and (5) a very high ratio of TP force to PImax was associated with a reduced propensity to moderate-to-severe OSA. 

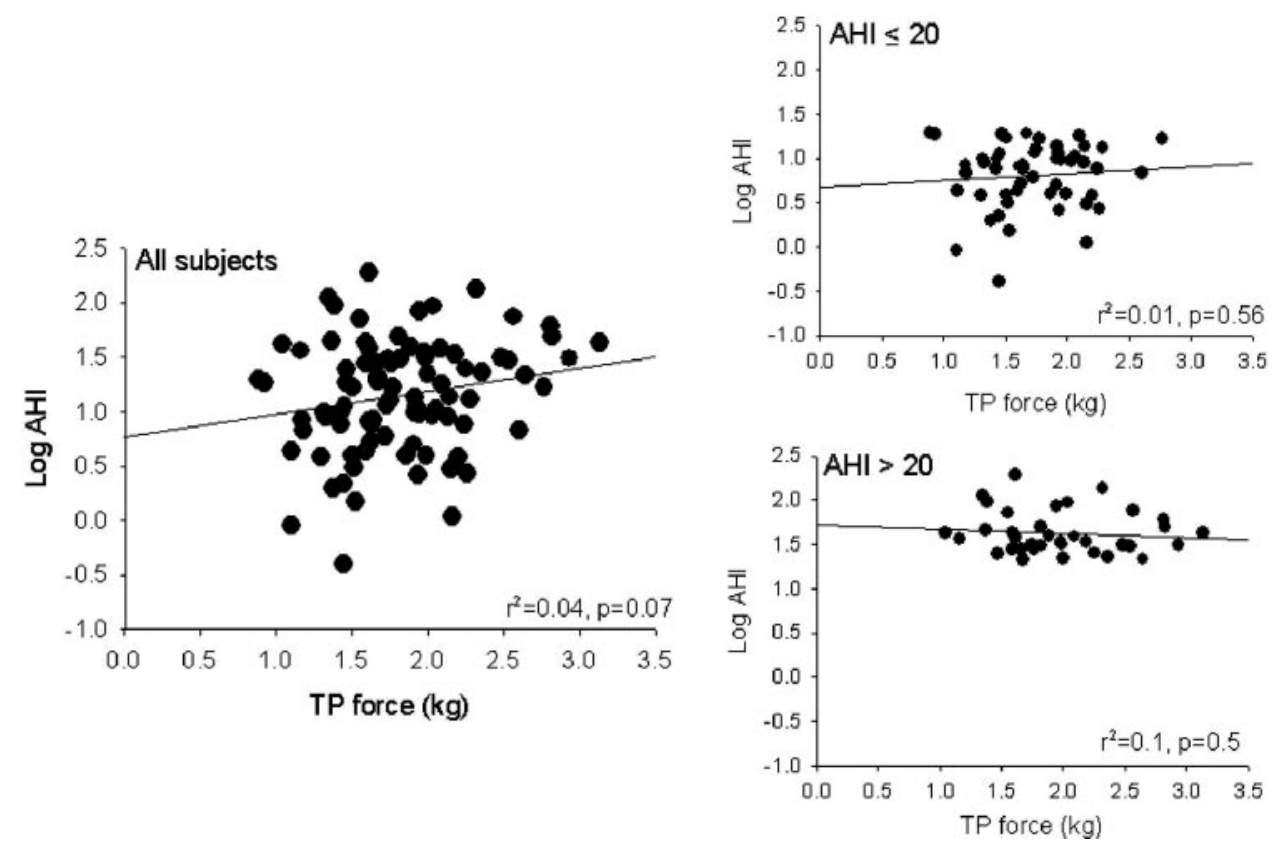

FIGURE 1. Relationship between TP force (measured seated) and severity of OSA (log AHI) in all subjects $(\mathrm{n}=94)[$ left] and when separated into those without OSA (AHI $\leq 20 / \mathrm{h}, \mathrm{n}=52)$ [top right] and with OSA (AHI $>20 / \mathrm{h}, \mathrm{n}=42$ ) [bottom right]. The solid lines represent regression lines.

\section{UA Muscle Strength and OSA}

There are several reasons why waking measurements of TP force may reflect the capacity of the dilator muscles to maintain airway patency during sleep. Firstly, the genioglossus is the major dilator muscle of the UA, ${ }^{1,17}$ being primarily responsible for maintenance of pharyngeal patency. Secondly, it has previously been shown that genioglossus electromyogram activity is related to TP strength; thus, a measure of TP force should reflect its maximal capacity to dilate the UA. ${ }^{18}$ Thirdly, the genioglossus
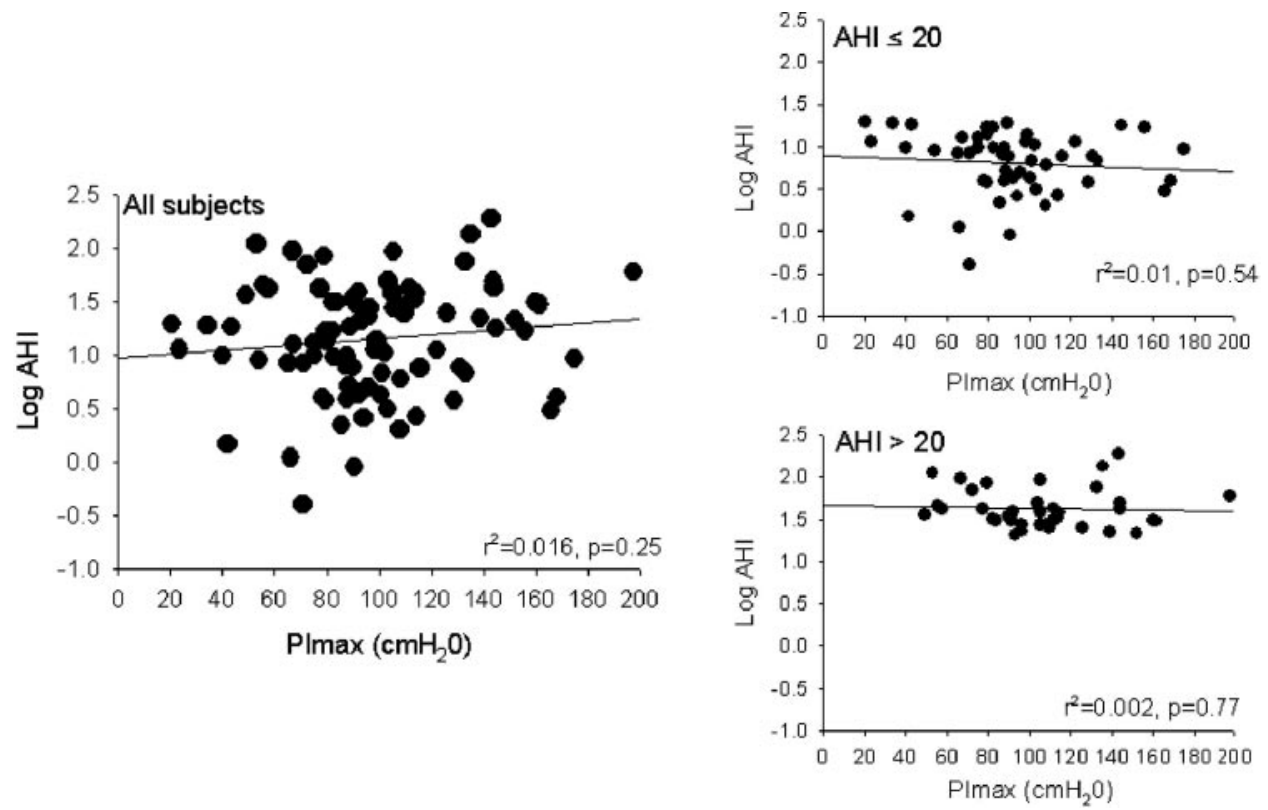

FIgURE 2. Relationship between PImax-FRC and severity of OSA ( $\log$ AHI) in all subjects $(\mathrm{n}=94)$ $[l e f t]$ and when separated into those without OSA (AHI $\leq 20 / \mathrm{h}, \mathrm{n}=52)[$ top right $]$ and with OSA (AHI $>20 / \mathrm{h}, \mathrm{n}=42$ ) [bottom right]. The solid lines represent regression lines. 

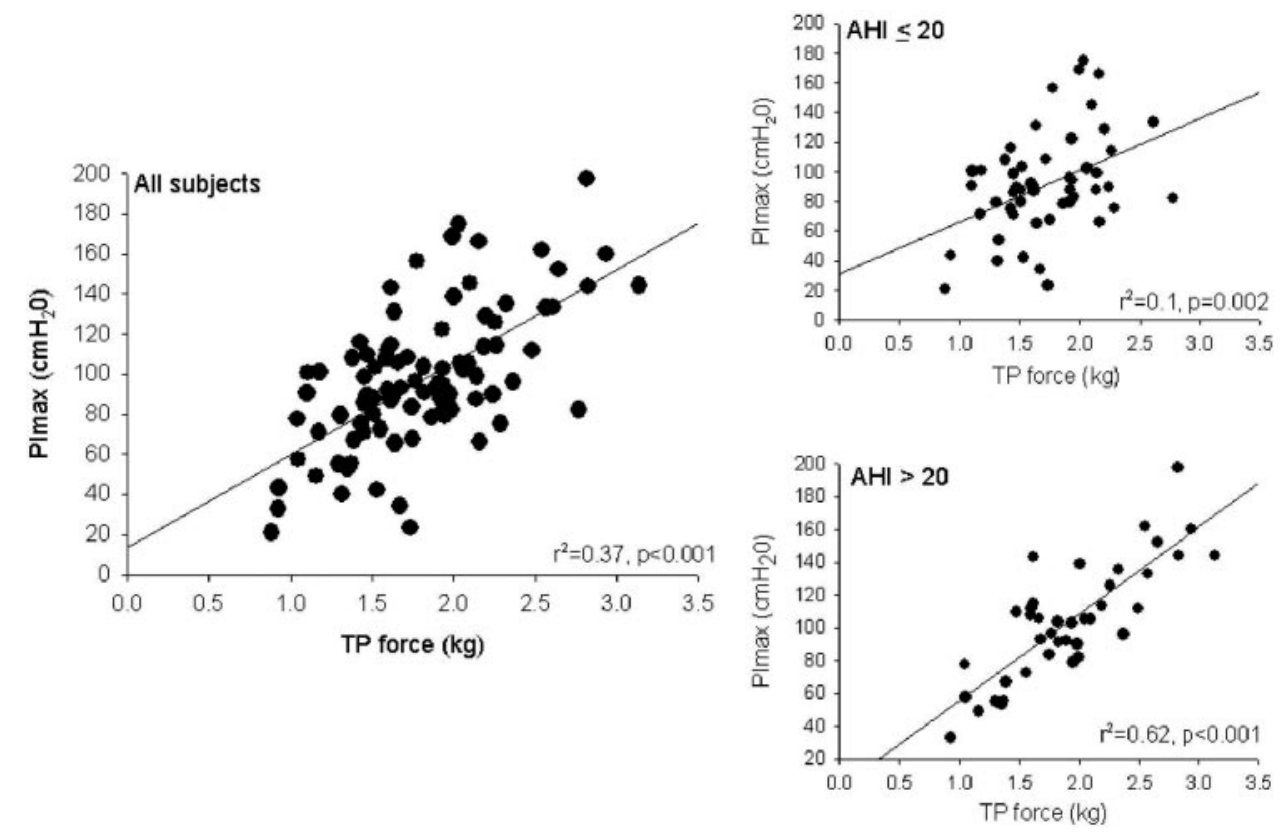

Figure 3. TP force (measured seated) vs PImax-FRC in all subjects $(\mathrm{n}=94)[\mathrm{left}]$ and when separated into those without OSA (AHI $\leq 20 / \mathrm{h}, \mathrm{n}=52$ ) [top right] and with OSA (AHI $>20 / \mathrm{h}, \mathrm{n}=42$ ) [bottom right]. The solid lines represent regression lines.

muscle has been shown to have an increased proportion of type II muscle fibers and an increase in anaerobic enzyme markers in patients with OSA, compared to normal individuals and nonapneic snorers. ${ }^{3,5}$ Such changes are consistent with an increased capacity of the UA muscles to produce force, ${ }^{3}$ which could be reflected in wakeful, voluntary maximal efforts. Fourthly, studies have shown that training the genioglossus muscle with electrical stimulation during wakefulness can decrease snoring, ${ }^{19,20}$ improve symptoms of daytime sleepiness, and decrease AHI. ${ }^{20}$

The present study found that supine TP force was significantly greater in individuals with moderate-tosevere OSA; however, such a difference was not

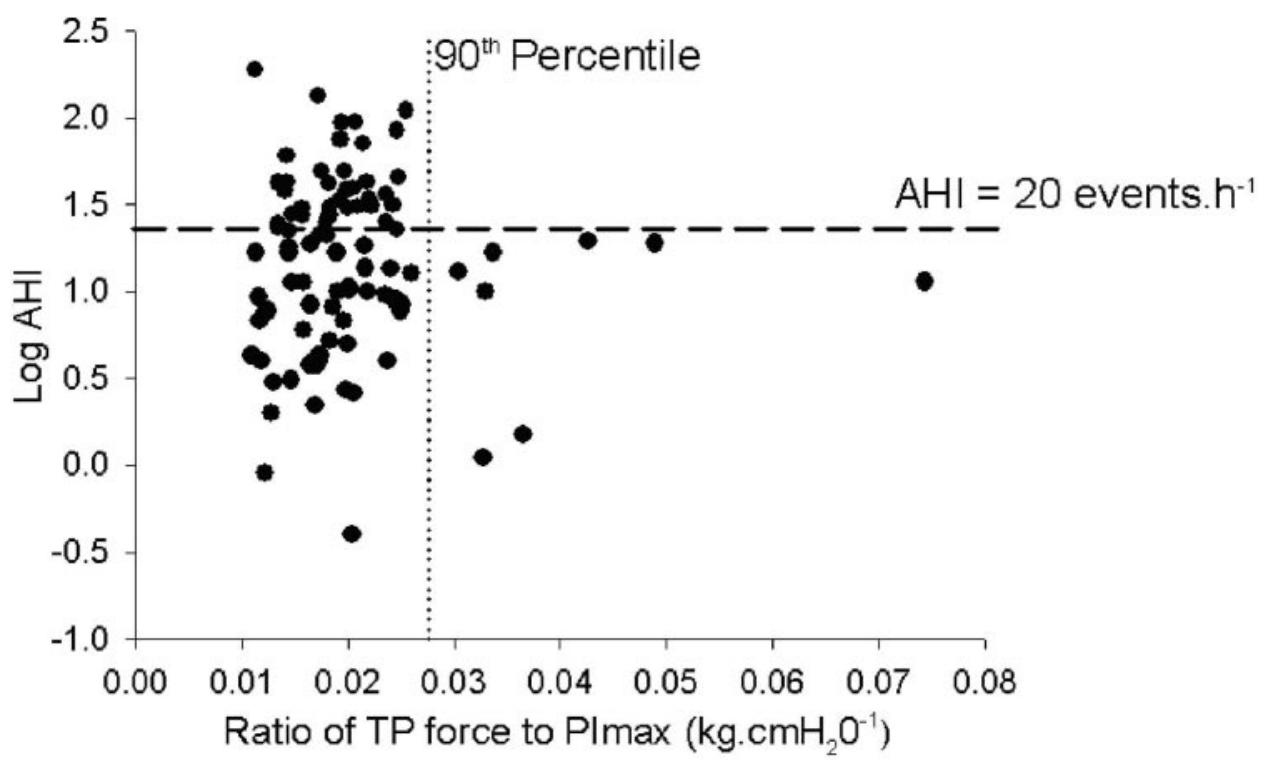

FIgURE 4. Ratio of TP force to PImax vs log AHI. Black circles represent all individuals. Data are presented with TP force measured in the seated position and PImax-FRC. The dashed line represents an AHI of 20/h. The dotted line represents the 90th around the median. 
apparent when measurements of TP force were controlled for age and BMI. This most likely reflects the observation that TP force decreases with increasing age and BMI. ${ }^{7}$ The lack of relationship between $\log$ AHI and TP force in our group of subjects and the weak correlation between these measures reported by Mortimore et $\mathrm{al}^{7}$ argue against an effect of OSA on the capacity of the UA muscles to generate force. ${ }^{5}$ While such a conclusion differs to studies ${ }^{3-5}$ showing an increase in type II muscle fibers and an increase in in vitro twitch tension in UA muscle fibers from OSA patients, ${ }^{3}$ the weak and variable nature of these relationships reported in the present and other studies ${ }^{3-5,7}$ argue against a major training effect on the UA muscles as a result of repetitive overnight muscle activation in response to UA narrowing in OSA. Further, they suggest that any abnormal structural changes in UA muscles reported in individuals with $\mathrm{OSA}^{3-5}$ do not have a major influence on the capacity of the tongue to produce force. The clinical significance of these structural changes in relation to OSA remains unknown.

\section{Respiratory Pump Muscle Strength and OSA}

To date, no study has specifically examined the relationship between respiratory pump muscle strength and the severity of OSA. It is possible that the strength of the diaphragm and other inspiratory muscles could be increased in patients with OSA by virtue of the "training effect" of repetitive overnight inspiratory efforts against an occluded or narrowed airway. Alternatively, it is possible that in obese subjects in whom the risk of OSA is increased, ${ }^{21}$ a decrease in chest wall compliance ${ }^{10}$ could impair the force-generating capacity of the inspiratory muscles. However, against the latter possibility we found no relationship between absolute or percentage of predicted PImax and BMI in our subjects, consistent with findings of an earlier study. ${ }^{22}$

The present study found no difference in PImaxFRC or PImax-RV between individuals with and without OSA, and no relationship between PImax and $\log$ AHI. These results suggest that OSA has no overnight training effect on respiratory pump muscles but contrast with those of Mezzanotte et al, ${ }^{23}$ who measured PImax in 14 OSA subjects and 11 normal control subjects and reported a significantly higher PImax in the control group. The reasons for the disparate findings are unclear but may relate to between-study differences in sample size, characteristics of the control group, and methodology.

\section{Ratio of UA Muscle Strength and Pump Muscle Strength}

The notion of the importance to UA patency of the balance between genioglossal force tending to open the airway and inspiratory muscle force tending to close it through generation of negative intrapharyngeal pressure has been extant since the seminal work of Remmers et al. ${ }^{1}$ Our findings suggest that relative force-generating capacity of these muscles is important, with relatively strong UA muscles protecting against moderate-to-severe OSA. It is unclear whether this is the result of cause or consequence. However, our findings of a lack of association between TP force and AHI, PImax and AHI, and the finding of a linear association between $\mathrm{TP}$ force and PImax suggests that any change in force-generating capacity of the UA dilator and inspiratory muscles is an inherent characteristic rather than acquired as a consequence of OSA.

Other factors may have played a role in the lack of association between AHI and TP force, PImax, or the ratio of TP force to PImax. Because measures of TP force and PImax are reliant on subject effort, it is possible that they were underestimated in some individuals. However, we believe this unlikely, as subjects were highly motivated, strongly encouraged, and the results reproducible, differing by no more than $5 \%$ over a minimum of three efforts. Further, in the case of Pimax, group means were $>100 \%$ of the predicted value. We chose to measure PImax in the seated position because the effect of supine position on PImax is known, ${ }^{24}$ and we were keen to make the measurements in a condition that best simulated how it would be used clinically (ie, seated). Because the effect of posture on TP force was unknown, we measured it both seated and supine. While it could be argued that it would be more appropriate to compare measurements of TP force and PImax supine, as it is in the posture that we sleep, it is likely that the strong association between $\mathrm{TP}$ force and Pimax observed when seated would be replicated when supine. While it could also be argued that the lack of association between AHI and TP force, PImax, or the ratio of TP force to PImax reflects the fact that AHI is measured during sleep while the latter measures are made during wakefulness, this is by necessity as wakefulness is a necessary condition to assess maximum voluntary strength.

In conclusion, while there was no direct relationship between the ratio of UA muscle strength (TP force) to inspiratory pump muscle strength (PImax) and AHI, it was notable that moderate-to-severe OSA was not observed in any individual with a ratio exceeding the 90th percentile for the group $(0.027$ $\mathrm{kg} / \mathrm{cm} \mathrm{H}_{2} \mathrm{O}$ ), suggesting that a very high waking ratio may indeed be protective of UA collapse during sleep.

ACKNOWLEDGMENT: We wish to thank the technical staff of the West Australian Sleep Disorders Research Institute for their support. 


\section{REFERENCES}

1 Remmers JE, DeGroot WJ, Sauerland EK, et al. Pathogenesis of upper airway occlusion during sleep. J Appl Physiol 1978; 44:931-938

2 Santos C, Braghiroli A, Mazzini L, et al. Sleep-related breathing disorders in amyotrophic lateral sclerosis. Monaldi Arch Chest Dis 2003; 59:160-165

3 Series F, Cote C, Simoneau JA, et al. Physiologic, metabolic, and muscle fiber type characteristics of musculus uvulae in sleep apnea hypopnea syndrome and in snorers. J Clin Invest $1995 ; 95: 20-25$

4 Smirne S, Iannaccone S, Ferini-Strambi L, et al. Muscle fibre type and habitual snoring. Lancet 1991; 337:597-599

5 Carrera M, Barbe F, Sauleda J, et al. Patients with obstructive sleep apnea exhibit genioglossus dysfunction that is normalized after treatment with continuous positive airway pressure. Am J Respir Crit Care Med 1999; 159:1960-1966

6 Boyd JH, Petrof BJ, Hamid Q, et al. Upper airway muscle inflammation and denervation changes in obstructive sleep apnea. Am J Respir Crit Care Med 2004; 170:541-546

7 Mortimore IL, Bennett SP, Douglas NJ. Tongue protrusion strength and fatigability: relationship to apnoea/hypopnoea index and age. J Sleep Res 2000; 9:389-393

8 Weiner P, Waizman J, Weiner M, et al. Influence of excessive weight loss after gastroplasty for morbid obesity on respiratory muscle performance. Thorax 1998; 53:39-42

9 Yap JC, Watson RA, Gilbey S, et al. Effects of posture on respiratory mechanics in obesity. J Appl Physiol 1995; 79: $1199-1205$

10 Naimark A, Cherniack RM. Compliance of the respiratory system and its components in health and obesity. J Appl Physiol 1960; 15:377-382

11 Mortimore IL, Fiddes P, Stephens S, et al. Tongue protrusion force and fatigability in male and female subjects. Eur Respir J 1999; 14:191-195

12 Rechtschaffen A, Kales A. A manual of standardized terminology, technique and scoring system for sleep stages of human sleep. Washington, DC: National Institutes of Health, 1968
13 Sleep-related breathing disorders in adults: recommendations for syndrome definition and measurement techniques in clinical research: the report of an American Academy of Sleep Medicine Task Force. Sleep 1999; 22:667-689

14 He J, Kryger MH, Zorick FJ, et al. Mortality and apnea index in obstructive sleep apnea: experience in 385 male patients. Chest 1988; 94:9-14

15 Hamilton AL, Killian KJ, Summers E, et al. Muscle strength, symptom intensity, and exercise capacity in patients with cardiorespiratory disorders. Am J Respir Crit Care Med 1995; 152:2021-2031

16 Black LF, Hyatt RE. Maximal respiratory pressures: normal values and relationship to age and sex. Am Rev Respir Dis 1969; 99:696-702

17 Sauerland EK, Mitchell SP. Electromyographic activity of intrinsic and extrinsic muscles of the human tongue. Tex Rep Biol Med 1975; 33:444-455

18 Mortimore IL, Douglas NJ. Relationship of genioglossus muscle electromyographic activity to force. Am J Respir Crit Care Med 1996; 153:A690

19 Randerath WJ, Galetke W, Domanski U, et al. Tonguemuscle training by intraoral electrical neurostimulation in patients with obstructive sleep apnea. Sleep 2004; 27:254259

20 Verse T, Schwalb J, Hormann K, et al. Submental transcutaneous electrical stimulation for obstructive sleep apnea [in German]. HNO 2003; 51:966-970

21 Kyzer S, Charuzi I. Obstructive sleep apnea in the obese. World J Surg 1998; 22:998-1001

22 Fiz JA, Aguilar X, Carreres A, et al. Postural variation of the maximum inspiratory and expiratory pressures in obese patients. Int J Obes 1991; 15:655-659

23 Mezzanotte WS, Tangel DJ, White DP. Waking genioglossal electromyogram in sleep apnea patients versus normal controls (a neuromuscular compensatory mechanism). J Clin Invest 1992; 89:1571-1579

24 Koulouris N, Mulvey DA, Laroche CM, et al. The effect of posture and abdominal binding on respiratory pressures. Eur Respir J 1989; 2:961-965 


\section{Relationship Between Upper Airway and Inspiratory Pump Muscle}

Force in Obstructive Sleep Apnea

Kelly L. Shepherd, Cathryn M. Jensen, Kathleen J. Maddison, David R. Hillman and Peter R. Eastwood Chest 2006;130;1757-1764

DOI 10.1378/chest.130.6.1757

This information is current as of November 12, 2008

\section{Updated Information \& Services}

References

Open Access

Permissions \& Licensing

Reprints

Email alerting service
Updated information and services, including high-resolution figures, can be found at: http://chestjournal.org/cgi/content/full/130/6/1757

This article cites 22 articles, 9 of which you can access for free at:

http://chestjournal.org/cgi/content/full/130/6/1757\#BIBL

Freely available online through CHEST open access option

Information about reproducing this article in parts (figures, tables) or in its entirety can be found online at: http://chestjournal.org/misc/reprints.shtml

Information about ordering reprints can be found online: http://chestjournal.org/misc/reprints.shtml

Receive free email alerts when new articles cite this article sign up in the box at the top right corner of the online article.

Images in PowerPoint format Figures that appear in CHEST articles can be downloaded for teaching purposes in PowerPoint slide format. See any online article figure for directions.

A M E R I C A N C O L L E G E O F

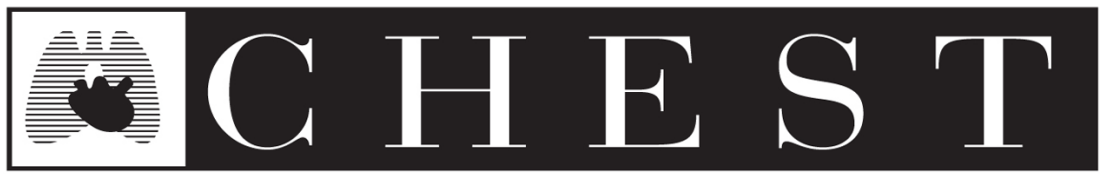

$\begin{array}{lllllllllll}\mathrm{P} & \mathrm{H} & \mathrm{Y} & \mathrm{S} & \mathrm{I} & \mathrm{C} & \mathrm{I} & \mathrm{A} & \mathrm{N} & \mathrm{S}^{\circledR}\end{array}$

